\title{
Various Modalities of Mandibular Defects Reconstruction: Overview
}

Dr. Bhavan Chand Yemineni ${ }^{1 *}$, Dr. Soumyasnata Maiti ${ }^{2}$, Dr. Abhijit Datta ${ }^{3}$, Dr. Syed Vizarat $\mathrm{Ali}^{4}$, Dr. Vaibhav Singh Thakur $^{4}$, Dr. Rishabh Harish Jain ${ }^{5}$

${ }^{1}$ Associate Professor, Department of Dental and Oral Surgery, ASRAM Medical College, Eluru, Andhra Pradesh, India

${ }^{2}$ PG Student, OMFS, KLE Belgaum

${ }^{3}$ PG Student, OMFS, Divya Jyoti College of Dental Sciences and Research, Ajit Mahal, Modinagar - Niwari Rd, Modinagar, Uttar Pradesh 201204, India

${ }^{4}$ PG Student, OMFS, Maharana Pratap College of Dentistry \& Research Centre, Gwalior, India

${ }^{5}$ PG Student, OMFS, ACPM Dental College, Dhule, Maharashtra, India

DOI: $10.36348 / \mathrm{sjm} .2020 . \mathrm{v} 05 \mathrm{i} 03.001$

| Received: 16.02 .2020 | Accepted: 01.03.2020 | Published: 06.03.2020

*Corresponding author: Dr. Bhavan Chand Yemineni

Abstract

Mandibular reconstruction is a complex procedure and is a challenge to the surgeon. Reconstruction is required following resection of mandible due to benign or malignant pathology, osteonecrotic mandible that could not be treated conservatively. Reconstruction modalities have changed significantly over the years with the introduction of newer techniques and technologies. This article reviews and summarizes the various reconstructive modalities for mandibular defects of past years, comparatively newer reconstructive options available and the recent advances in reconstruction done by Patient-Specific Implants produced by virtual planning.

Keywords: Mandible, Defects, Reconstruction.

Copyright @ 2020: This is an open-access article distributed under the terms of the Creative Commons Attribution license which permits unrestricted use, distribution, and reproduction in any medium for non-commercial use (NonCommercial, or CC-BY-NC) provided the original author and source are credited.

\section{INTRODUCTION}

Oromandibular reconstruction resulting from resection of benign tumour, malignant tumor, and osteomyelitis or osteoradionecrotic mandible remains a challenge for the surgeon today [1]. After resection the patient would be left with considerable hard and soft tissue deficit which mandates reconstruction not only to replace the missing structural component, but also to restore the associated function. This restoration of form and the function becomes more and more difficult as the tissues resected become larger and complex in nature [2]. Following resection, mandibular defects require a multidisciplinary approach to optimize functional and cosmetic outcomes. Comprehensive reconstructive strategies require the restoration of facial dimensions, including width, height, and projection. To achieve optimal functional and aesthetic results, reconstructive surgeons must be able to replace the skeletal buttresses, restore the external/internal soft tissue envelope, eliminate fistulas, and provide a foundation for dental rehabilitation [1]. Morphologically the mandible defines the contour of the lower third of the face [3] and the geometric design of the inferior border of the mandible defines the aesthetic contour of the lower third of the face. This horizontal buttress or mandibular plane defines a soft tissue cephalometric parameter formed by a line that connects menton to gonion [1]. The U-shaped mandible serves as the arch of the oral cavity, supporting the tongue and muscles of the mouth floor, thus permitting mastication, articulation, deglutition, and respiration. These days tumour surgery of maxillofacial region demands not just a radical removal of a tumour with primary or secondary reconstruction. Furthermore, the patient requires the full function and dental rehabilitation [1]. The evolution of better diagnostics, technology and understanding of surgical sciences, there is shift towards functional procedures which spare maximum tissue as possible. Reconstructive modalities have also largely increased in recent years that gives possibility to restore back maximum function and aesthetics. In this article we review various reconstructive modalities for mandibular defects including the history and recent advances in reconstruction done using $\mathrm{CAD} / \mathrm{CAM}$ which could serve as the treatment of choice for mandibular reconstruction.

\section{HISTORY}

Medical literature can be seen dating back to 1860 detailing the concept of mandibular reconstruction. With the initial experimental sciences giving way to clinical application of free grafts from tibia, iliac crest and ribs used for small to large defects, 
extensive work has been done. Mainstay of mandibular reconstruction at the inception remained the use of autogenous bone grafts [2]. Bardenheur and skyoff described the use of free autologous bone grafts for mandibular reconstruction $[4,5]$. Martin described the reconstruction of a resected mandibular segment with a prosthetic appliance [6]. Metal band was used for mandibular defect reconstruction by Partsch [7]. Berndt recommended the use of celluloid material. Silver wire was used to bridge the gap after resection of a portion of the lower jaw by White in a 9 year old boy [8]. Metals have also been used; Vitallium, Stainless steel and Titanium [9]. Leuke and Rappaport, Schwartz and Albert and associates used Dacron urethane mesh for holding the cancellous chips [10]. The introduction of internal fixation using plate and screws post World War II with the use of antibiotics gave surgeons an unparalleled edge over these procedures [2]. Much has been written over the years about multistaged techniques employing pedicled and even free soft tissue flaps supporting a neomandibular scaffold. These flaps isolate the contaminated oral cavity and at second surgeries are filled with cancellous bone grafts from the iliac crest with or without bone morphogenic proteins. Once new bone forms, osseointegrated implants are placed at a third surgery. The resulting neomandible can be an excellent replacement for the missing mandible segment, but the time is long and the costs are high .Immediate reconstruction of the missing mandible with vascularized bone and placement of osseointegrated implants during a single anesthesia has proven to be more reliable and much more cost effective. What has evolved is the choice of which free osseous or osteocutaneous flap to employ [3]. Taylor et al. described the iliac crest microvascular free flap for just this reconstruction in 1979 [10]. The flap was reliable and the bone stock could be harvested to closely mimic the mandible in size and shape. The 1980s saw the introduction of the radial forearm flap incorporating a partial thickness segment of the radius and scapula osteocutaneous flap employing the lateral border of the scapula [3]. The fibula flap was also introduced by Taylor et al. in 1975 for long bone reconstruction [11]. Bradley in 1978 and 1982 reported a two-stage procedure for reimplantation of 'autogenous freeze treated mandibular bone '[12]. The first-in-human study involving the use of BoneMorphongenic Proteins (rhBMP) for mandibular reconstruction was performed in 2001 by Moghadam. For defects with extensive hard and soft tissue loss, microvascular free tissue transfer often provides an excellent reconstructive option [13].

\section{Etiology}

Acquired segmental defects of the mandible are most commonly secondary to ablative tumor therapy or avulsive traumatic injury. Other less common causes include inflammatory or infectious conditions that result in devitalisation of the mandibular bone requiring its debridement [3]. Reconstruction of mandibular defects is usually performed immediately at the time of benign tumor ablation (e.g., ameloblastoma), malignant cancer extirpation, or the resection of osteomyelitic and stage III osteoradionecrotic mandible. Primary repair of oromandibular defects offers significant advantage over secondary repair by preventing the wound from scarring while obtaining optimal functional and aesthetic results for the patient [14]. Management of oral squamous cell carcinoma is the most common malignancy resulting in acquired segmental defects of the mandible. Avulsive segmental wounds most commonly arise from highvelocity injuries such as firearms, industrial accidents, and occasionally motor vehicle collisions [1]. Amount of destruction of soft and hard tissue depends upon kinetic energy associated with the injury. Loss of tissue increases dramatically as the speed of the missile or the speed of impact increases as $(\mathrm{KE}=\mathrm{mv} 2)$.

\section{Goals of Reconstruction}

The primary goals of oromandibular reconstruction are to achieve primary wound closure as well as to obtain a functional and aesthetic restoration [14]. Further, goals of mandibular reconstruction are to re-establish the form of the lower third of the face and to restore the patient's ability to eat in public, be intelligible to both trained and untrained listeners, and to maintain an unencumbered airway that allows the freedom to perform all activities. An ideal reconstruction of a mandibular defect would need the replacement of structural bony foundation, restoration of the supporting muscle and soft tissue envelope, and provide a platform for dental rehabilitation post reconstruction [2]. Reconstruction should establish continuity, alveolar height, arch form, arch width and Improve facial contours [1].

\section{Classification of Mandibuar Defects}

The defect determines the kind of reconstruction needed and Classification of defects gives a more precise description and allows us to choose from various reconstructive options available for each patient and defect [2]. Since Pavlov's classification of mandibular defects was first published in 1974, six additional classification systems have been proposed. The most widely cited classification system is the HCL classification by Jewer and colleagues [15]. The Jewer classification provides an aid in classifying mandibular defects [16] and reflects the complexity of the reconstructive problem. Central defects including both canines are designated ' $\mathrm{C}$,', and lateral segments that exclude the condyle are designated ' $\mathrm{L}$.' 'When the condyle is resected together with the lateral mandible, the defect is designated ' $\mathrm{H}$,' or hemimandibular. Eight permutations of these capital letters- C, L, H, LC, HC, LCL, HCL, and $\mathrm{HH}$ - are encountered for mandibular defects. 


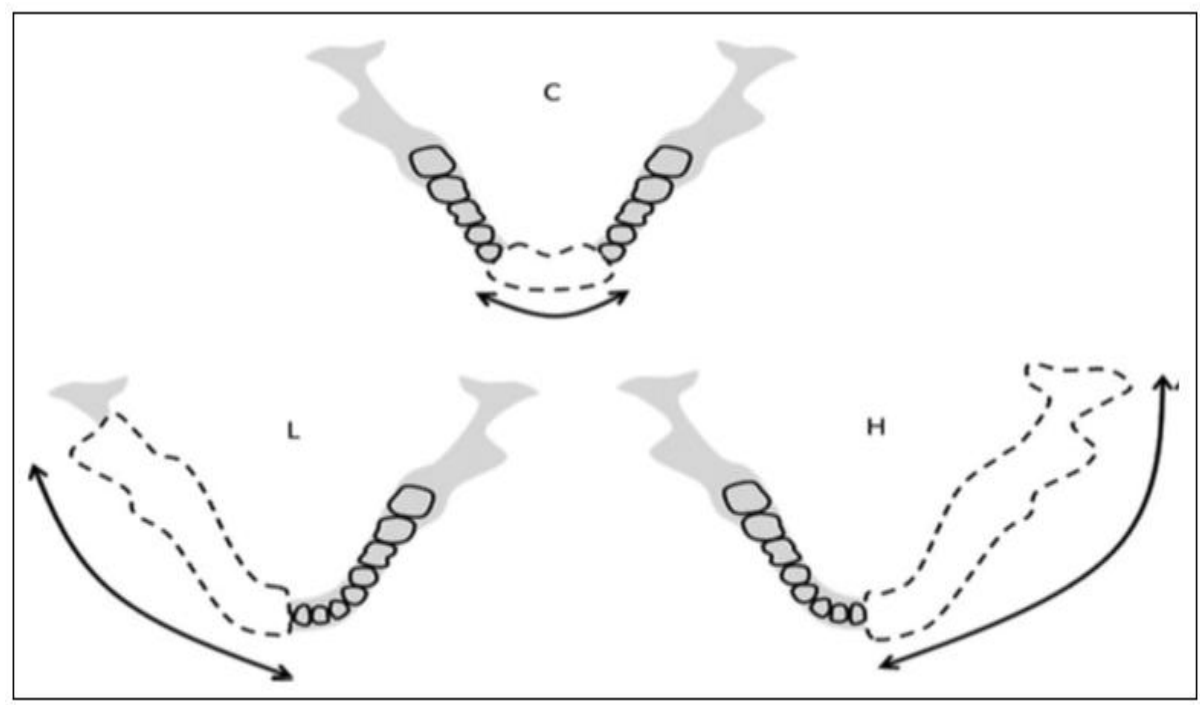

Fig-1: Taken from- Mandibular Reconstruction: Overview

\begin{tabular}{|c|c|}
\hline & Classification \\
\hline Pavlov $^{1}$ & $\begin{array}{l}\text { By the number of remaining bone fragments: class } \mid=\text { one bone } \\
\text { fragment, class } I I=\text { two bone fragments, class }|I|=\text { three bone } \\
\text { fragments }\end{array}$ \\
\hline David et al ${ }^{4}$ & $\begin{array}{l}\mathrm{A}=\text { lateral, } \mathrm{B}=\text { unilateral angle to symphysis, } \mathrm{C}=\text { angle and body of } \\
\text { other side, } \mathrm{D}=\text { angle to angle, } \mathrm{E}=\text { symphysis, } \mathrm{F}=\text { hemimandible } \\
\text { including condyle }\end{array}$ \\
\hline Jewer et $\mathrm{al}^{2}$ & $\begin{array}{l}\mathrm{H}=\text { unilateral condyle but can cross midline, } \mathrm{L}=\text { unilateral no } \\
\text { condyle but can cross midline, } \mathrm{C}=\text { both canines, } \mathrm{HC}=\text { lateral and } \\
\text { condyle including both canines, } \mathrm{LC}=\text { lateral and both canines, } \\
\mathrm{LCL}=\text { bilateral lateral defects including canines but not condyles, } \\
\mathrm{HCL}=\text { condyle lateral, central, and controlateral lateral, } \\
\mathrm{HCH}=\text { entire mandible. }\end{array}$ \\
\hline Urken et aß & $\begin{array}{l}\mathrm{C}=\text { condyle, } \mathrm{R}=\text { ramus, } \mathrm{B}=\text { body, } \mathrm{S}=\text { symphysis, } \mathrm{S}^{\mathrm{H}}=\text { stops at the } \\
\text { midline }\end{array}$ \\
\hline lizuka et $\mathrm{al}^{6}$ & Class IN based on the number of osteotomies of the fibula flap \\
\hline Hashikawa et al ${ }^{\mathrm{S}}$ & $\begin{array}{l}\mathrm{C}=\text { loss of condylar head, } \mathrm{A}=\text { loss of angle, } \mathrm{T}=\text { loss of mental } \\
\text { tubercle, } \mathrm{CAT}=\text { hemimandible }\end{array}$ \\
\hline Baumann et al & $\begin{array}{l}\text { Type l=segment not including condyle, type } \| \text { =segment including } \\
\text { condyle (specifically for osteoradionecrosis) }\end{array}$ \\
\hline
\end{tabular}

Fig-2: Taken from - A new classification for mandibular defects after oncological resection

According to James S Brown, Conor Barry, Michael Ho, and Richard Shaw A new classification system of mandibular defects based on the four corners of the mandible is suggested [15].

\section{Reconstruction Modalities}

Bone reconstruction should replace the missing segment of mandible while maintaining the proper alignment of the remaining native mandible in order to minimize problems with mouth opening and malocclusion [17]. The best functional and aesthetic results occur with immediate mandible reconstruction. Delayed reconstruction results in scarring and fibrosis of the remaining bone and soft tissue, making the proper placement of the reconstructed bone rather difficult or even impossible [18].

\section{Comparatively Newer Modalities Avascular Bone Grafts}

Nonvascularized autogenous bone grafts can be used for reconstruction of small to medium size mandibular defects. These can be harvested from the patients scull, rib, ilium, tibia, fibula, scapula, humerus, radius, and metatarsal bones which can provide viable and immunocompatible osteoblastic cells [17]. The bone graft is placed in a well vascularized bed. The adjacent bone fragments are stripped of periosteum so that adequate bone to bone contact is established [1]. The iliac crest and the rib are the most common donor sites. The primary drawback to these stem from the extensive and rapid resorption where up to $60 \%$ of the grafted volume is lost within six months [2]. 


\section{Vascularized Pedicled Bone Transfer}

In 1980' was developed the use of pectoralis major and latissimus dorsi muscles as pedicled myocutaneous flaps that were transferred with the segment of underlying fifth rib [19]. The pectoralis major myocutaneous (PMMC) flap is a widely used workhorse in head and neck reconstruction, first used on thoracic defects by Pickrell in 1947 [2]. It is reliable and relatively easy to harvest, features a large volumeof muscle and subcutaneous tissue and can fill large defectsobliterating dead spaces in reconstruction. LD provides a large amount of muscle and cutaneous lining with minimal donor site morbidity.

\section{Microvascular Osteocutaneous Free Flaps}

Microvascular free tissue transfer has been one of the greatest milestones in reconstruction of the mandible after tumour ablative surgery popularized by hidalgo in 1989 paper [19]. The tissue should be of sufficient length, width and height for reconstruction of a proposed defect and should be well vascularized with a pedicle of adequate length [20]. It is the most reliable method to achieve single- stage, immediate reconstruction of the mandible and are considered the gold standard untill the new methods utilizing vascularized tissue engineered mandibular grafts are developed .

\section{Transport Disc Distraction Osteogenesis (TDDO)}

For mandibular reconstruction, a technique known as transport disc distraction osteogenesis (TDDO) is used. A segment of bone is cut adjacent to the defect and moved gradually across the defect by a mechanical device. New bone fills in between the two bone segments. Transport distraction for reconstruction of continuity defects is most efficient for defects of the mandibular body. When used to reconstruct a defect of the body of the mandible, the transported segment not only achieves bone continuity but also, through histiogenesis, the associated attached tissue is reconstructed achieving a natural ridge with a vestibule [1]. Osteogenetic distraction has some risks such as infection, loosening of the distractor, paraesthesia, and excessive skin damage caused by the pins leading to facial scar as the inevitable result of the extraoral device [17].

\section{Tissue Engineering}

Tissue engineering is an interdisciplinary field that combines the principles of engineering, material and biological sciences toward the development of therapeutic strategies and biological substitutes that restore, maintain, replace or improve biological functions [1]. Growth factors and smad proteins, such as bone morphogenic proteins, and platelet rich plasma osteoprogenitor cells produced on a recipient bed can induce adequate regeneration. The development of bone morphogenic proteins (BMPs) has offered an alternative to traditional bone grafting, which has been the gold standard for oral and maxillofacial reconstruction Many growth factors are involved in osteogenesis. Bone morphogenetic proteins (BMP-2 and BMP-7), transforming growth factor beta (TGF- $\beta$ ), insulin-like growth factors I and II (IGF I and II), platelet-derived growth factor (PDGF), fibroblast growth factors (FGFs), and vascular endothelial growth factor (VEGF) [13]

\section{Alloplastic Materials \\ Mandibular reconstruction plates and screws are the most widely used alloplastic devices for mandibular reconstruction. Commonly used metals used in the fabrication of these plates are stainless steel, vitallium and titanium. Stainless steel and titanium reconstruction plates were a mandibular reconstructive option that is fast, single-staged and reliable while maintaining oral function and form. Shortcomings are the adverse reaction of alloplastic, non-biologic materials [17].}

\section{Recent Advances \\ Patient - Specific Implants (PSI)}

The use of individualized designed biomaterials has opened new possibilities in reconstructive surgery, and by the use of patientspecific computed tomography (CT), it is possible to use files to construct patient-specific implants (PSIs) [22]. Precious time in the operating room is invested in plate contouring to reconstruct the mandible. Rapid prototyping technologies can construct physical models from computer-aided design via 3-dimensional (3D) printers. A prefabricated 3D model is achieved, which assists in accurate contouring of plates and/or planning of bone graft harvest geometry before surgery. The 2 most commonly used rapid prototyping technologies are STEREOLITHOGRAPHY AND 3D PRINTING (3DP). Three-dimensional printing is advantageous to stereo lithography for better accuracy, quicker printing time, and lower cost. Rapid prototyping is a class of technologies that can construct physical models from computer-aided design via $3 \mathrm{D}$ printers [21, 22]. A transfer key method (TK-method) was developed by Frank Wilde (2015) where plates were pre-bent using a patient specific mandible model and positioned on the mandible with the help of transfer keys. The "standard method" (ST-method) and A transfer key method (TKmethod) were compared in a clinical trial. The TKmethod was more accurate than the ST method in a clinical trial. The TK-method was effective and accurate for mandible reconstruction using pre-bent fixation plates. 

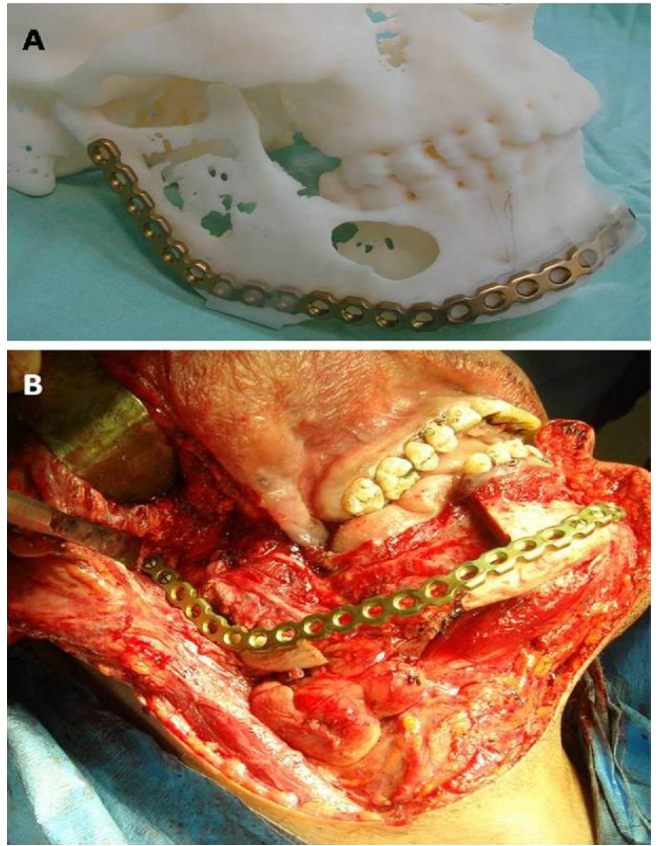

Fig-3(a)(b): Taken from - Mandibular reconstruction using stereolithographic 3-dimensional printing modeling technology

\section{Mandibular Reconstruction Using ProPlan CMF}

ProPlan CMF (Depuy Synthes, Solothurn, Switzerland, and Materialise, Leuven, Belgium) is a computer-aided surgical virtual planning service with professionalmedical engineers, and transfers patientspecific surgical guides to the virtual plan. Currently published reports are very limited due to a small number of cases and short-term follow-up. Large-scale and long-term follow-up studies are demanded [23].

\section{CONCLUSION}

Reconstruction modalities for mandibular defects has evolved over many years. Various newer modalities has been introduced but ideal reconstruction is still a challenge for surgeons. Demand for newer techniques with more precision has introduced patient specific implants that saves time of a surgeon during surgery. These recent advances in reconstruction modalities combines surgical procedures with virtual planning. The advantages of the $3 \mathrm{D}$ model techniques include the special understanding of the bone morphology, an accurate and easier planning of plate preoperative. Preoperative bending, and a much more accurate bone harvesting by using the negative imprint of the gap to be reconstructed. Hopefully this evolution of reconstruction option from autogenous bone grafts, $\mathrm{K}$-wire to 3D Printing modeling will bring revolution in the field of surgery and in future this 3D Printing technology will bring ease and precision in other fields of Surgery.

\section{REFERENCES}

1. Kumar, B. P., Venkatesh, V., Kumar, K. J., Yadav, B. Y., \& Mohan, S. R. (2016). Mandibular reconstruction: overview. Journal of maxillofacial and oral surgery, 15(4), 425-441.
2. Pai, D., Wodeyar, A., Raja, P., Nishad, M., Martis, E., \& Kumar, K. (2019). Evolution of mandibular defects reconstruction procedures: from older principles to newer techniques and technology. Acta Scientific Dent Sci, 3(5):8-18.

3. Baccarani, A., \& De Santis, G. (2015). Mandible Reconstruction: A Review Article. Journal Surgery, S(1):7.

4. IVY, R. H. (1951). Bone grafting for restoration of defects of the mandible. Plastic and Reconstructive Surgery, 7(4), 333-341.

5. Tessier, P., Kawamoto, H., Matthews, D., Posnick, J., Raulo, Y., Tulasne, J. F., \& Wolfe, S. A. (2005). Taking long rib grafts for facial reconstruction-tools and techniques: III. A 2900case experience in maxillofacial and craniofacial surgery. Plastic and reconstructive surgery, 116(5), 38S-46S.

6. Testelin, S. (1992, June). History of microsurgical reconstruction of the mandible. In Annales de chirurgie plastique et esthétique, 37(3):241-245.

7. Defries, H. O. (1981). Reconstruction of the mandible: use of combined homologous mandible and autologous bone. Otolaryngology--Head and Neck Surgery, 89(4), 694-697.

8. White, S. (1909). The employment of silver wire to bridge the gap after resection of a portion of the lower jaw. British medical journal, 2(2552), 1525.

9. Attie, J. N., Catania, A., \& Ripstein, C. B. (1953). A stainless steel mesh prosthesis for immediate replacement of the hemimandible. Surgery, 33(5), 712-720.

10. Albert, T. W., Smith, J. D., Everts, E. C., \& Cook, T. A. (1980). Dacron mesh tray and cancellous bone in reconstruction of mandibular defects. Archives of Otolaryngology-Head \& Neck Surgery, 8:78-83.

11. Taylor, G. I., Miller, G. D., \& Ham, F. J. (1975). The free vascularized bone graft. A clinical extension of microvascular techniques. Plastic and reconstructive surgery, 55(5), 533-544.

12. Bradley, P. F. (1982). A two-stage procedure for reimplantation of autogenous freeze-treated mandibular bone. Journal of Oral and Maxillofacial Surgery, 40(5), 278-284.

13. Herford, A. S., Stoffella, E., \& Tandon, R. (2011). Reconstruction of mandibular defects using bone morphogenic protein: can growth factors replace the need for autologous bone grafts? A systematic review of the literature. Plastic surgery international, 2011.

14. Lin, P. Y., Lin, K. C., \& Jeng, S. F. (2011). Oromandibular reconstruction: the history, operative options and strategies, and our experience. ISRN surgery, 2011.

15. Brown, J. S., Barry, C., Ho, M., \& Shaw, R. (2016). A new classification for mandibular defects after oncological resection. The Lancet Oncology, 17(1), e23-e30. 
16. Jewer, D. D., Boyd, J. B., Manktelow, R. T., Zuker, R. M., Rosen, I. B., Gullane, P. J., ... \& Freeman, J. E. (1989). Orofacial and mandibular reconstruction with the iliac crest free flap: a review of 60 cases and a new method of classification. Plastic and reconstructive surgery, 84(3), 391-403.

17. Stošić, S. (2000). Mandibular reconstruction state of the art and perspectives surgery. Semin Surg Oncol, 19(3):226-234.

18. Disa, J. J., \& Cordeiro, P. G. (2000, October). Mandible reconstruction with microvascular surgery. In Seminars in surgical oncology, 19(3):226-234. New York: John Wiley \& Sons, Inc.

19. Hidalgo, D. A. (2008). Fibula Free Flap Mandible Reconstruction, Military Medical Academy, Clinic for Maxillofacial Surgery. Belgrade, 65(5):397403.

20. Schuepbach, J., Dassonville, O., Poissonnet, G., \& Demard, F. (2007). Early postoperative bone scintigraphy in the evaluation of microvascular bone grafts in head and neck reconstruction. Head \& face medicine, 3(1), 20.

21. Cohen, A., Laviv, A., Berman, P., Nashef, R., \& Abu-Tair, J. (2009). Mandibular reconstruction using stereolithographic 3-dimensional printing modeling technology. Oral Surgery, Oral Medicine, Oral Pathology, Oral Radiology, and Endodontology, 108(5), 661-666.

22. Wilde, F., Winter, K., Kletsch, K., Lorenz, K., \& Schramm, A. (2015). Mandible reconstruction using patient-specific pre-bent reconstruction plates: comparison of standard and transfer key methods. International journal of computer assisted radiology and surgery, 10(2), 129-140.

23. Sasaki, R., \& Rasse, M. (2017) Mandibular Reconstruction Using ProPlan CMF: A Review. Craniomaxillofac Trauma Reconstruction Open, 1:e35-e42. 